6. Hulscher ME, Laurant MG, Grol RP. Process evaluation on quality improvement interventions. Qual Saf Health Care 2003; 12:40-46.

7. Baskerville N, Bruce MHA, Hogg W, Lemelin J. Process evaluation of a tailored multifaceted approach to changing family physician practice patterns improving preventive care. J Fam Pract 2001; 50:242-249.

8. Kritchevsky SB, Braun BI, Wong ES, et al. Impact of hospital care on incidence of bloodstream infection: the evaluation of processes and indicators in infection control study. Emerg Infect Dis 2001; 7:193-196.

\section{Catheter-Associated Bloodstream Infections in 2 Long-Term Acute Care Hospitals}

TO THE EDITOR-Catheter-associated bloodstream infections (BSIs) are a well-known source of excess morbidity and mortality.' The National Nosocomial Infections Surveillance (NNIS) System Report contains data on the rates of catheter-associated BSI that occur in intensive care units (ICUs) in US hospitals. ${ }^{2}$ This information is important for establishing comparison rates that facilities can use to set thresholds for intervention. However, there are no comparable data available on catheter-associated BSI rates for longterm acute care hospitals (LTACHs).

An LTACH is defined by the Center for Medicare Services as a hospital that has an average inpatient length of stay of greater than 25 days. The number of LTACHs in the United States has grown significantly in the past decade, and there are currently more than 275 of these facilities. ${ }^{3}$ LTACHs provide extended medical and rehabilitative care for clinically complex patients, including patients with multiple organ dysfunction, ${ }^{4}$ and those who require prolonged weaning from mechanical ventilation. One recent study demonstrated that rates of antimicrobial resistance and device use in LTACHs are comparable to those seen in ICUs. ${ }^{5}$

The purpose of our study was to determine the central lineassociated BSI rate for a cohort of patients with respiratory failure who were admitted to $2 \mathrm{LTACH}$ for ventilator weaning and to compare this rate to rates determined by the NNIS for ICUSs in the United States. Data were collected from November 2004 through July 2005 as part of a prospective, observational study in 2 LTACHs. One LTACH had 12 beds, the second had 30. Catheter-associated BSI was defined according to Centers for Disease Control and Prevention criteria. ${ }^{6}$ To ensure that only infections acquired in LTACHs were included, we excluded infections with symptom onset within 48 hours after admission. Approval from the institutional review board of Emory University was obtained for this study, and written consent was obtained from all subjects.

Data were collected for 93 patients. The total number of patient-days was 2,685, and the total number of central linedays was 2,007 , yielding a central line use rate of $74.7 \%$. This rate is equivalent to the 90th percentile of central line use rates for medical and combined medical-surgical units re- ported in the latest NNIS report. ${ }^{2}$ There were 33 BSIs during the study period, yielding a central line-associated BSI rate of 16.44 cases per 1,000 central line-days. This is higher than the reported 90th percentile rate for ICUs of all types and is roughly double the 90th percentile rate reported for medical and medical-surgical units. ${ }^{2}$ Enterococci and coagulase-negative staphylococci were the most common pathogens recovered from blood cultures (Table). Staphylococcus aureus and Candida species each accounted for $12 \%$ of the total number of isolates.

It is possible that our data are skewed toward higher rates because all of the patients admitted to our facilities had a primary admission diagnosis of respiratory failure. Hence, our patients may be sicker than the overall LTACH population. However, the median length of stay among our patients (24 days) was comparable to or shorter than those reported in other studies. ${ }^{5,7}$ Our data may also reflect suboptimal catheter insertion and care techniques. Much progress has been made in recent years to improve these practices in short-term acute care hospitals. LTACHs are a comparatively new care setting, and hence these best practices for catheter insertion and care may not yet be widely implemented.

Because patients had acute illnesses and prolonged lengths of stay, we hypothesized that the rates of catheter-associated BSI in LTACHs would be comparable to those seen in ICUs. In fact, we found that catheter-associated BSI rates at 2 LTACHs exceeded the 90th percentile of rates for all ICUs reporting data to the NNIS system. The rate of 16.44 cases per 1,000 central line-days was approximately double the 90th percentile rate for medical and combined medical-surgical ICUs, the units whose patient populations most closely resemble those of LTACHs. The majority of organisms recovered were gram-positive bacteria; more than $10 \%$ of these BSIs were cases of fungemia. Our findings demonstrate the need for more data on rates of catheter-associated BSIs in LTACHs. This information, collected from a variety of LTACH types, will allow for comparison of infection rates between LTACHs and will help determine thresholds for interventions. Though limited by a small sample size and by possible se-

TA B LE. Pathogens Isolated From 33 Patients With Catheter-associated Bloodstream Infections

\begin{tabular}{lc}
\hline Organism & $\begin{array}{c}\text { No. (\%) } \\
\text { of isolates } \\
(N=40)\end{array}$ \\
\hline Enterococcus species & $13(32)$ \\
Coagulase-negative staphylococci & $12(29)$ \\
Staphylococcus aureus & $5(12)$ \\
Candida species & $5(12)$ \\
Klebsiella oxytoca & $3(8)$ \\
Acinetobacter baumannii & $1(3)$ \\
Alcaligenes xylosoxidans & $1(3)$ \\
\hline
\end{tabular}

a Some patients had 2 organisms recovered. 
lection bias, our data suggest that catheter-associated BSIs are a significant problem in LTACHs. Further work is needed to characterize infection rates better and to design interventions to prevent BSIs in LTACHs.

\section{ACKNOWLEDGMENTS}

This study was supported by a grant from the Carlyle Fraser Heart Center Foundation.

Linda L. Wolfenden, MD; Grant Anderson, BA; Emir Veledar, PhD; Arjun Srinivasan, MD

From the Divisions of Pulmonary, Allergy and Critical Care Medicine (L.L.W.), Cardiology (E.V.), and Infectious Diseases (A.S.), and the School of Medicine (G.A.), Emory University, and the Centers for Disease Control and Prevention (A.S.), Atlanta, Georgia.

Address reprint requests to Linda L. Wolfenden, MD, Emory University, Division of Pulmonary, Allergy and Critical Care Medicine, 1365A Clifton Road, 4th floor, Atlanta, GA 30322 (1wolfen@emory.edu).

Infect Control Hosp Epidemiol 2007; 28:105-106

(C) 2006 by The Society for Healthcare Epidemiology of America. All rights reserved. 0899-823X/2007/2801-0022\$15.00.

\section{REFEREN CES}

1. Pittet D, Tarara D, Wenzel RP. Nosocomial bloodstream infection in critically ill patients. Excess length of stay, extra costs, and attributable mortality. JAMA 1994; 271:1598-1601.

2. National Nosocomial Infections Surveillance (NNIS) System Report, data summary from January 1992 through June 2004, issued October 2004. Am J Infect Control 2004; 32:470-485.

3. Liu K, Baseggio C, Wissoker D, Maxwell S, Haley J, Long S. Long-term care hospitals under Medicare: facility-level characteristics. Health Care Financ Rev 2001; 23:1-18.

4. Dematte D'Amico JE, Donnelly HK, Mutlu GM, Feinglass J, Jovanovic BD, Ndukwu IM. Risk assessment for inpatient survival in the long-term acute care setting after prolonged critical illness. Chest 2003; 124:1039-1045.

5. Gould CV, Steinberg JP. Antibiotic resistance in long-term acute care hospitals (LTACHs): the perfect storm. Infect Control Hosp Epidemiol 2006; 27:920-925.

6. Garner JS, Jarvis WR, Emori TG, Horan TC, Hughes JM.CDC definitions for nosocomial infections, 1988. Am J Infect Control 1988; 16:128-140.

7. Bach PB, Carson SS, Leff A. Outcomes and resource utilization for patients with prolonged critical illness managed by university-based or community-based subspecialists. Am J Respir Crit Care Med 1998; 158:1410-1415.

\section{Behavioral Explanation of Noncompliance With Hand Hygiene}

We have read with great interest the article by Dr. Whitby and colleagues on a behavioral explanation for noncompliance with hand hygiene. ${ }^{1}$ Compliance with hand hygiene has improved as a result of using alcohol-based hand rub, but still rarely exceeds $60 \%$ under study conditions. Therefore, new approaches and methods such as those suggested by Dr. Whitby are required to reach high rates of compliance. However, several issues have not yet been clearly addressed. The authors used focus group discussions to collect information, rather than applying written questionnaires. The results of these discussions are not given as percentages of positive or negative answers to questions, but as individual responses from participants. For example, Whitby et al. note that "mothers and nurses agreed that hand washing in the home is of lesser importance," ${ }^{(1446)}$ and community attitudes toward hand washing are presented in Table 2. The statements in Table 2 are likely individual responses from select participants, but not a representative view of all participants.

Data from the focus groups were then used to create a questionnaire. Nurses were asked to complete this questionnaire, but the results are not given in detail. Sixty-one percent of the nurses responded to the questionnaire; no data were available on the reasons for nonparticipation. In most studies, some questions are not answered, and such data should be given to estimate the potential of a bias in this study.

Whitby et al. assert that "translation of community handwashing behavior to healthcare settings is the predominant driver of all handwashing...."1(p484) This statement may be true in countries where hand washing with soap and water is the standard of care in healthcare institutions, but may not be applicable to countries in Europe, where use of alcohol-based hand rub is the standard of care in healthcare institutions. Since alcohol-based hand rub is rarely used in European households, the translation from community hand washing standards to the use of alcohol-based hand rub in healthcare institutions in such countries may not be applicable as it might be in Australia.

Whitby et al. ${ }^{1}$ state that only a small increase in adherence to hand hygiene guidelines may be seen after introduction of alcohol-based hand rub. In many other studies, sufficient time to perform hand hygiene was the key factor in improving compliance $\mathrm{e}^{2-4}$; likewise, staff shortage was a risk factor for transmission of pathogens. ${ }^{5,6}$

We congratulate Whitby et al. ${ }^{1}$ on their efforts to expand current techniques to improve adherence with components derived from the "Theory of Planned Behavior." However, the results of their study should not impede or delay implementing the use of alcohol-based hand rub in institutions where hand washing with soap and water is still the standard of care. In addition, behavior changes in healthcare institutions require tremendous effort, a long-standing commitment, and special education of trainers. The change from hand washing with soap and water to the use of alcoholbased hand rub will increase compliance, and the transition should not be postponed because of the lack of a concomitant behavior modification program. It also would impede the Word Health Organization efforts in 2006 to promote the use of alcohol-based hand rub as the standard of care. ${ }^{6}$

Andreas Widmer, MD 